\title{
Zur Tal- und Reliefgeschichte des Churfirsten-Alvier-Gebietes (Kanton St. Gallen)
}

\begin{abstract}
:
The valley and relief history of the Churfirsten-Alvier range (Ct. St. Gallen):

Valley formation in the Churfirsten-Alvier range, between the Linth and Rhine rivers, began at the end of the Miocene with the tectonic history of the Helvetic nappes. In early stages, the Cretaceaous sequences became independent of their Jurassic substratums and these from their Verrucano-Triassic basement. The valley of Lake Walenstadt in the south was initiated between this basement and north-moving younger sequences. The Thur valley, north of the Churfirsten range. which follows a syncline in the highest Helvetic nappe, was filled with early Tertiary sediments and Pennine Flysch. The valleys in the Alvier area are formed along synclines and faults in the Cretaceous sequence; these structures dip towards the Rhine valley.

The Churfirsten range forms a limestone shield in the Helvetic nappe. It broke up following $\pm \mathrm{N} / \mathrm{S}$-oriented joints. There inbetween, excavation of kars (cirques) began already during pre-Quaternary glaciation; they are still intact at the ends of the range, but in the middle the kar-walls broke down along the steeper southern slope.

During the Mindel and Riss glaciations, no Rhine ice flowed through the saddles between the Churfirsten peaks into the Thur valley; however, this could have been possible in preMindelian cold periods at high ice levels and still lower mountain relief.
\end{abstract}

\section{Key words:}

Pliocene, Pleistocene, Reliefgeschichte, cirques, Chürfirsten, Alvier, Ct. St. Gallen.

\section{Zur Erforschungsgeschichte}

Das Gebiet der Churfirsten-Alvier-Kette war wiederholt Gegenstand geologischer Untersuchungen. A. ESCHER v. d. Linth erarbeitete von 1835 bis 1864 die Grundlagen für eine erste geologische Karte 1:25000. 1869 zeichnete er eine Manuskript-Karte der St. Galler und Appenzeller Alpen, von der 1878 postum eine Säntis-Karte erschien.

A. LUDWIG $(1894,1897)$ faßte in den Churfirsten und im Alvier-Gebiet gemachte Beobachtungen mit kritischen Angaben zur Literatur (ESCHER et al. 1875K, MOESCH 1881 und Alb. HEIM 1891) berichtmäßig zusammen. Im Anschluß an die Churfirsten-Mattstock-Monographie (Arn. HEIM 1910-17) und die Aufnahmen zur Walensee-Karte (HEIM \& OBERHOL-
ZER 1907K) haben die beiden (1917K) auch das Alvier-Gebiet geologisch aufgenommen.

In neuerer Zeit haben THIERSTEIN (1969), BRIEGEL (1972), DIEGEL (1973), jüngst OUWEHAND (in FÖLLMI \& OUWEHAND. 1987) und GREBER (1987) die biostratigraphisch-faziellen Ergebnisse von GANZ (1910), HEIM (1910-17) und HEIM \& OBERHOLZER (1907K. 1917K) mikropaläontologisch und sedimentologisch-bathymetrisch ergänzt. BRIEGEL versuchte, eine Phasenfolge in der Bruchbildung aufzustellen. Nachdem das Gebiet auch quartärgeologisch untersucht worden ist (GUTZWILLER 1873, FREY 1916, MAU. RER 1952, HANTKE 1967, 1970, 1980), sei versucht. tal- und reliefgeschichtliche Aspekte - zusammen mit Abtragungsfragen - aufzudecken.

\section{Die helvetischen Decken: Ausscherung, Vormarsch, Platznahme}

Mit der Ausscherung von Sedimentabfolgen vom NRand des Ur-Mittelmeers, ihrem Vorgleiten auf geneigter Oberfläche und der Platznahme als osthelvetische Decken beginnt in der Seez-Walensee-Talung und im Churfirsten-Alvier-Gebiet die Tal- und Reliefgeschichte. Schon in Frühphasen hat sich dabei das Kreide-Stockwerk von seiner Jura-Unterlage gelöst und ist auf den mergelig-schieferigen Grenzschichten selbständig vorgefahren (HELBLING 1938). Für die Zeit des Abfahrens der Kreide-Hülle, die $\mathrm{N}$ der Wildhauser Mulde zu den in sich verscherten Falten des Alpstein hochgestaut worden ist. ergeben sich Hinweise aus den Molasse-Abfolgen. Diese reichen im Hörnli-Schuttfächer, den alpinen Schuttmassen eines erstmals vereinigten Bündner Rheins, bis ins jüngere Miozän. Im Napf-Gebiet, dem Schuttfächer einer Ur-Aare. deren Einzugsgebiet aufgrund des Geröll-Inhaltes weit nach S zurückgriff, endete die Schüttung bereits im mittleren Miozän. Für das Vorgleiten, die Stauchung und die endgültige Platznahme der helvetischen Decken verbleiben somit nur wenige Jahrmillionen. Beim Vorgleiten der Kreide wurde auch der von seiner

René Hantke, Prof., Dr., Geolog. Institut der ETH und der Universität Zürich, Sonneggstr. 5, CH-8092 Zürich 
Hülle entblößte Jura-Unterbau zu verfalteten Schuppen gestaucht.

Mit der Platznahme der helvetischen Decken waren die proximalen Schuttfächer-Stränge unterbrochen worden. Die aus den Alpen austretenden Entwässerungssysteme fanden neue Abflußwege, dabei umflossen sie die mit der veränderten Tektonik sich ergebenden Hindernisse.

Die Geschwindigkeit, mit der Deckenbewegungen erfolgt sind, läßt sich zwischen Val d'Illiez und Genfersee abschätzen; für die dort schon im mittleren Oligozän bewegten präalpinen Decken ergeben sich wenige Jahrmillionen oder $1 \mathrm{~cm} / \mathrm{a}$. Das letzte Vorgleiten der helvetischen Decken und der nachfolgende Hochstau des Aar-Massivs manifestieren sich besonders W der Verrucano-Kuppel, W der Linth. S der Klausenpaß-Linie hat sich von der überfahrenen Unterlage, verkehrtliegendem AxenLochseitenkalk, noch etwa $1 \mathrm{~km}^{2}$ erhalten, während sich vom Jura der darüber gefahrenen Axen-Decke nichts und von ihrer Trias nur bescheidene Rauhwacken-Reste erhalten haben. Analog ist weiter $N$, an der Pragelpaß-Linie, die Druesberg-Decke über die verkehrtliegende, als "Mittelschenkel» extrem ausgewalzte Toralp-Serie gefahren. Auf der Silberen-Decke, einer frontalen Abspaltung der AxenDecke, ist in einer Längsmulde gar ein Rest der Druesberg-Decke zurückgeblieben. Von ihr wie von der Axen-Decke sind an den südlichen Erosionsrändern nur wenige hundert Meter bis ein Kilometer abgetragen worden. Der Abtrag hat aber weder auf dem Lochseitenkalk noch auf derToralp-Serie über größere Bereiche haltgemacht; vielmehr bekunden diese Oberflächen Bewegungsflächen.

\section{Zur Geomorphogenese des Churfirsten-Alvier- Gebietes}

Die Anlage der Churfirsten-Alvier-Täler ist durch den tektonischen Bau der Gebirge zwischen Walensee, Rheintal und Toggenburg vorgezeichnet: im S durch die Südbegrenzung des Jura- und KreideStockwerkes, im E durch das Axialgefälle gegen das Rheintal und im $\mathrm{N}$ und $\mathrm{NW}$ durch die Wildhauser Mulde, die sich in Amdener und Flügenspitz-Mulde aufspaltet. In den Churfirsten und im Alvier-Gebiet wird sie durch den inneren Deckenbau und die daran beteiligten Störungen bestimmt; die bedeutendste, die Seez-Walensee-Talung, verdankt ihre Entstehung dem differentiellen Ab- und Vorgleiten des Malm/Kreide-Stockwerkes der MürtschenDecke, den aufgefahrenen Jura-Schuppen der Axen- und der Churfirsten-Decke sowie der Churfirsten-Alvier-Kreide. Zwischen dem S- bis SERand der vorgefahrenen Deckenteile und ihren zurückgebliebenen Trias/Lias-Kernen öffnete sich schon bei der Platznahme, im jüngsten Miozän, das Seez-Walensee-Tal.
In der Alvier-Kreide und in den gestauchten JuraKernen zeichnet sich ein markantes Achsengefälle gegen NE ab, so daß die Kreide zwischen Wartau und Grabs, bei Sevelen gar ihr jüngstes Schichtglied, die Wang-Formation, unter die Rhein-Alluvionen abtaucht. Rechtsrheinisch folgen darüber: Liechtensteiner und Vorarlberger Flysch, die penninische Falknis-Decke, die Aroser Zone und die ostalpine Lechtal-Decke. Damit ist auch die Ost-Seite, das Rheintal, analog begrenzt wie das Seeztal, jedoch nicht durch einen südlichen, sondern durch den westlichen Erosionsrand der penninischen und der ostalpinen Decken. Die penninischen Decken haben - wie die Grabser Klippen und die Gipfelklippen von Fäneren- und Flügenspitz belegen - sich weiter gegen Wfortgesetzt: von den ostalpinen Dekken jedoch fehlt in der NE-Schweiz jede Spur. Ihr Wiederauftreten über penninischen Elementen der östlichen Zentralschweiz erfolgte schon nach dem Ausscheren aus dem Ablagerungsraum, wohl durch eine weiter Wgelegene Depression.

Ursprünglich hingen die Churfirsten von der Amdener/Flügenspitz-Mulde über Leistchamm und die Firste bis zur Sichelchamm-Mulde als pultartiger Gebirgsrücken zusammen. Dies läßt sich zwischen Hinterrugg und Chäserrugg noch erkennen. Beidseits des Verbindungsgrates haben sich Kare ausgebildet. Auf der Nord-Seite, in der Chammeren, hat sich ein bis $1840 \mathrm{~m}$ absteigender Kar-Firn erhalten (Fig. 1). Im Valsloch bildete sich gar ein Zirkus auf der Süd-Seite; dagegen vermochte das W anschlieBende Kar den Grat nicht mehr zu erreichen (Fig. 2).

Die Ausräumung der Gesteinsfolgen scheint in der Churfirsten-Alvier-Kreide unterschiedlich tief erfolgt zu sein. In der die beiden trennenden Mulde, auf Galms NW des Sichelchamm, war sie bescheiden. Über dem Seewer Kalk sind noch 30 m Amdener Mergel erhalten. Die höheren Kreide- und Alttertiär-Abfolgen sind kaum vollständig erosiv abgetragen worden. Entweder sind sie gar nicht abgelagert worden, oder sie dürften auf Amdener Mergeln abgeglitten und schon bei der Bildung der Wildhauser Mulde in ihr verblieben sein.

In der axial zum Voralpsee abtauchenden Sichelchamm-Mulde werden die Amdener Mergel mächtiger. Der Nausbach hat sich schon als subglaziäre Schmelzwasserrinne tief in sie eingeschnitten. Auf der Nord-Abdachung der Churfirsten zwischen Selun und Hinterrugg/Oberruestel ist der Seewer Kalk bis auf kleine Relikte - auf der Breitenalp, der westlichen Selamatt und der Selunalp - abgetragen worden oder bereits in einer Frühphase auf den Fluebrig-Schichten abgefahren. Dabei kam - neben dem Zerbrechen der weitradig verbogenen Churfirsten-Decke - ein hoher Anteil der Lösung der Karbonate zu. Aufgrund vorhandener Mächtigkeiten muß für den Seewer Kalk im W mit mindestens 80 m, 


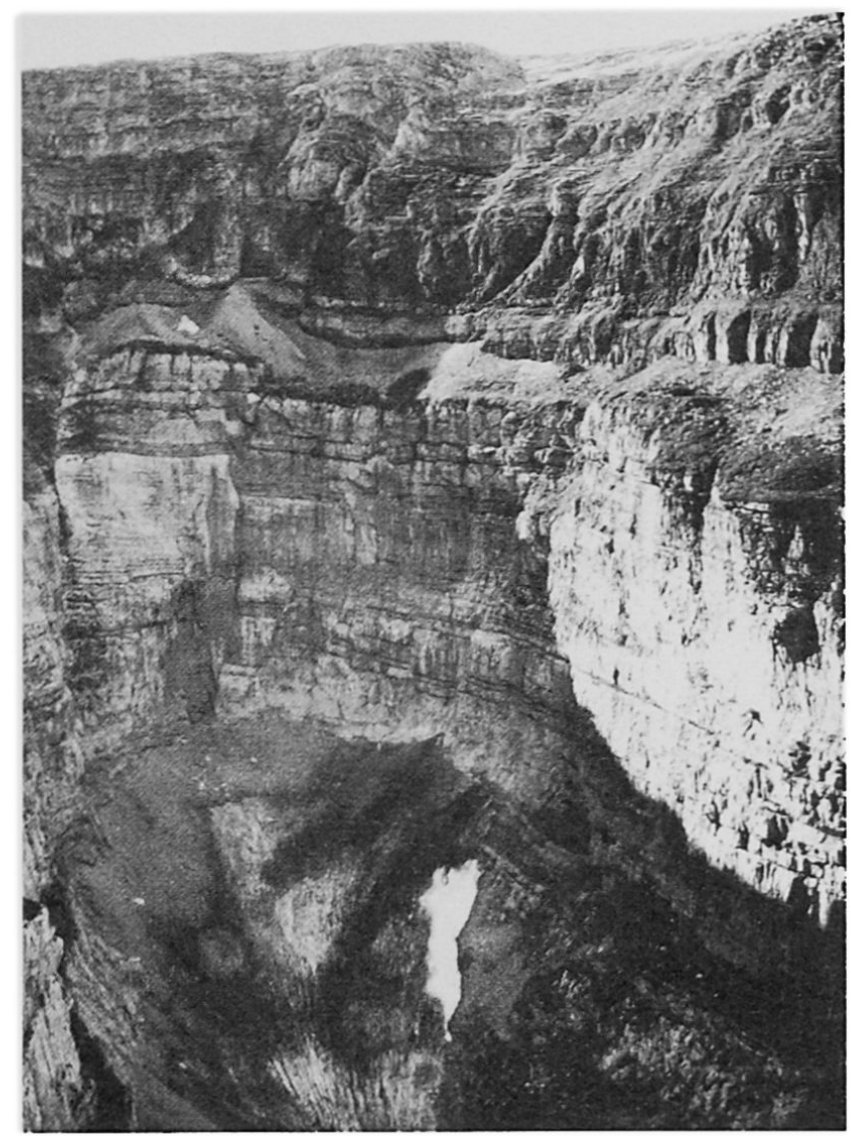

Fig. 1 N-exponiertes Churfirsten-Kar zwischen Chäserrugg und Hinterrugg (rechts) im Schrattenkaik, in der Garschella-Formation (= Gault, über der Bildmitte) und Seewer Kalk (oberes Viertel). Links eine letztspätglaziale Seitenmoräne.

im $\mathrm{E}$ gar mit über $125 \mathrm{~m}$ gerechnet werden. Bei der Annahme einer Abtragungsrate von $0,05 \mathrm{~mm} / \mathrm{a}$, was bei einer Höhenlage zwischen 1400 und $2300 \mathrm{~m}$ und einem Schichtfallen zwischen 0 und $45^{\circ}$ eher zu hoch sein dürfte, ergäbe sich ein Zeitraum zwischen 1,6 und 2,5 $\mathrm{M}$ a. Da der Abtrag zudem nicht an der Basis des Seewer Kalkes endet, sondern noch tief in die 60-80 m mächtige Garschella-Formation (Gault) und in den Karbecken gar bis $150 \mathrm{~m}$ in den Schrattenkalk hinuntergreift, anderseits lokal noch Reste von Amdener Mergel auf dem Seewer Kalk verblieben sind, ist dies als Minimal-Schätzung zu betrachten. Zudem wurden - vorab in Kar-Wannen durch vorstoßendes Eis - ganze Felspartien aus ihrem Verband ausgepreßt, am Eis festgefroren und talwärts verfrachtet. Von den Schrattenkalk-Erratikern auf der Nord-Abdachung der Churfirsten dürfte - trotz derTiefe der Kare - nur ein kleinerTeil auf diese Weise losgesprengt worden sein. Der Abtrag ist lokal sicher höher gewesen, doch fällt dies gesamthaft kaum ins Gewicht. Anderseits hat am Hinterrugg, am Chäserrugg, auf dem Rosenboden und am Tristelcholben ein mächtiger Seewer Kalk

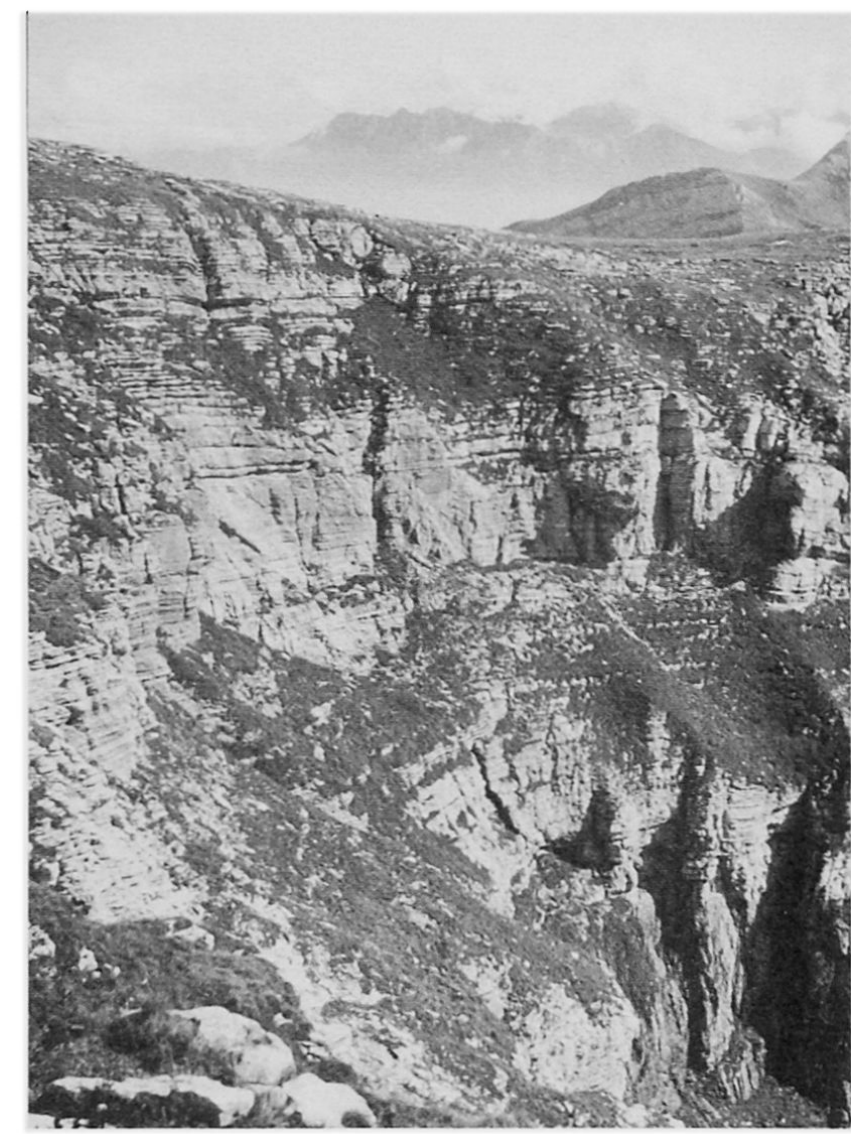

Fig. 2 S-exponiertes Churfirsten-Kar zwischen Chäserrugg und Hinterrugg (vorn) in der Garschella-Formation (= Gault, untere rechte Bildecke) und Seewer Kalk.

die Karbonatlösung überdauert, so daß vor allem der Zerscherung durch die bei der weitradigen Aufwölbung erzeugten Spannungen im Gesteinsverband, der Vergrößerung der Oberfläche und der längeren Einwirkung der Schmelzwässer Bedeutung zukommt. Ferner dürfte besonders dort, wo Seewer Kalk flach liegt - auf Hinterrugg und Chäserugg der Lösungsabtrag, wie in den Schwyzer Alpen mit $0,015 \mathrm{~mm} / \mathrm{a}$ (HANTKE 1982), geringer gewesen sein. Allenfalls kann gar eine geringe Bedeckung von nicht abgefahrenen Amdener Mergeln den Abtrag verzögert haben. Auf diese Möglichkeit deutet das muldenförmige Umbiegen des Seewer Kalkes auf der SE-Seite des Tristelcholben hin.

Die innere Gliederung der Churfirsten-AlvierKette in einzelne Gipfel und Grate und gegen N bzw. NE abfallende Täler ist damit tektonisch vorgezeichnet. Obwohl die Verstellungen bei den Bruchstörungen in den Churfirsten-Sätteln bescheiden sind (HEIM 1917), haben sie doch zur Bildung von Kar-Nischen beigetragen. HEIM (1917: 662) hat die halbrunden Felshohlformen noch nicht als echte Kare zu deuten gewagt, da an ihren Ausgängen die 

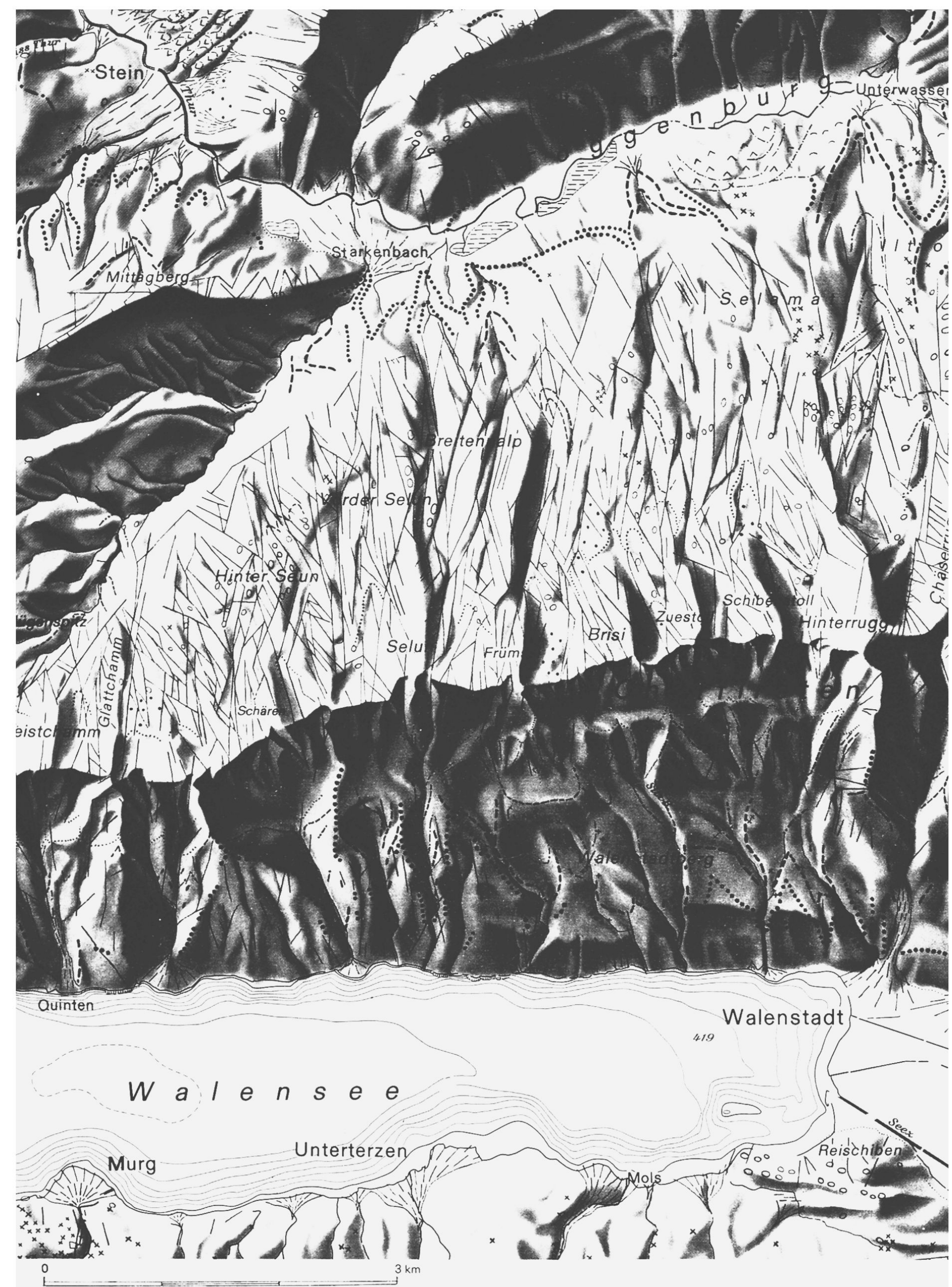

Den Herren Prof. E. Spiess, H. Stoll und A. Uhr sei für ihre Mithilfe bestens gedankt.

Reproduziert mit Bewilligung des Bundesamtes für Landestopographie vom 16. 12.1986. 


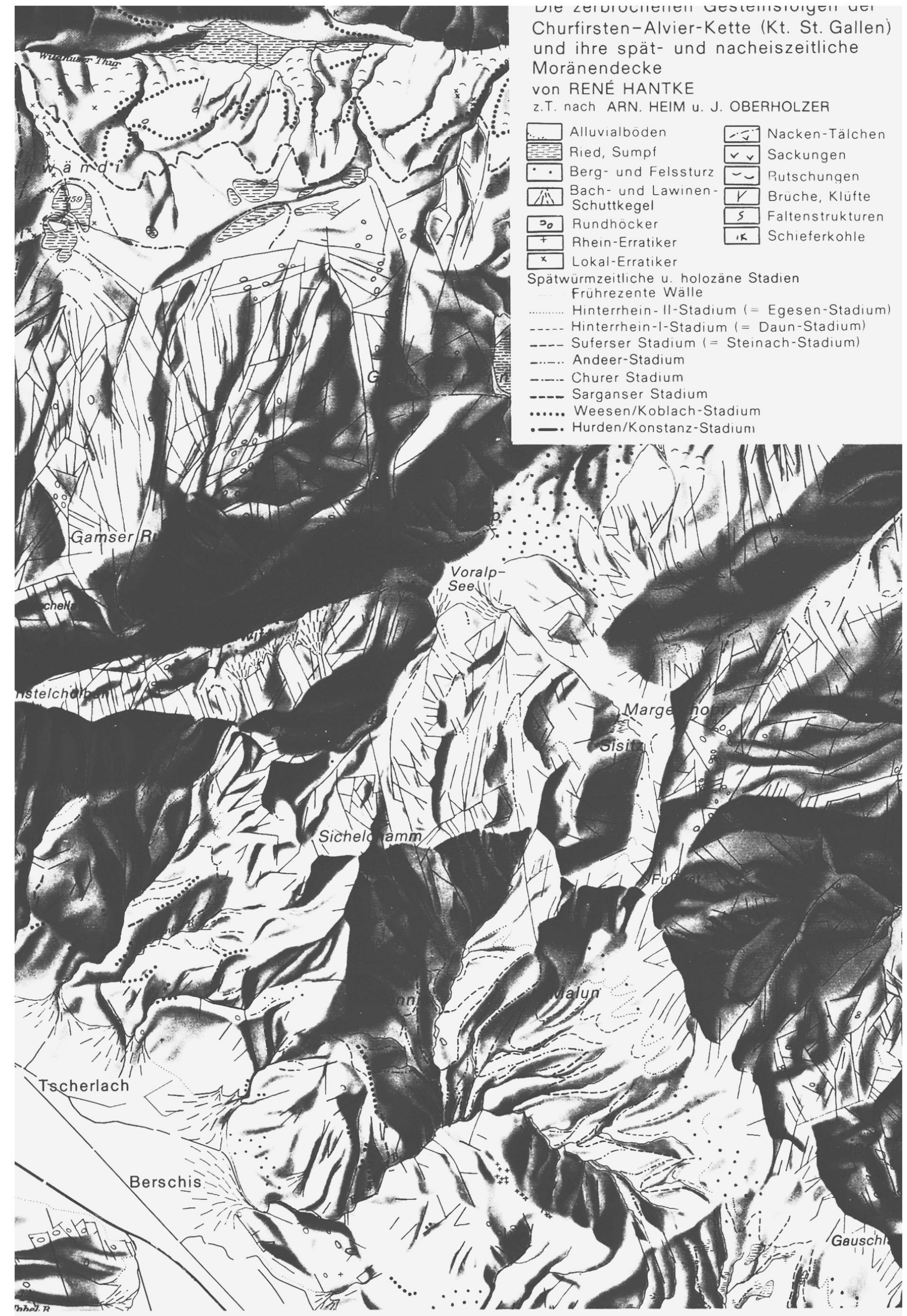


ausgeräumte Schuttmasse fehle. Da Schuttgut auf der N-Abdachung jedoch nur bescheiden auftritt, verblieb den Gletschern nur wenig für den Aufbau von Seiten- und Stirnmoränen, so daß die Wälle des mittleren und ausgehenden Spätwürms recht dürftig erscheinen. Offenbar haben aggressive Schmelzwässer die klüftigen Kalke der Hohlformen subglaziär aufgelöst und den Schutt einerseits in gelöster Form weggeführt; anderseits hat die Auskolkung weit länger gedauert, als allgemein angenommen worden ist.

Der steile Süd-Abfall der Churfirsten ist durch Frost-Einwirkung - mindestens von Vorder Säls bis Schrina-Obersäß - um wenige hundert Meter zurückgewittert. Damit wäre die ehemalige Kar-Rückwand - ausgenommen zwischen Chäserrugg und Hinterrugg, wo sie noch erhalten ist - gegen die Walensee-Seite niedergebrochen. Auch weiter W, zwischen Selun und Leistchamm, fehlt die Rückwand bei $\mathrm{N}$-orientierten Karen; nur zwischen Glattchamm und Leistchamm blieb sie intakt, so daß ein scharfer Grat die beiden verbindet (Karte).

Aus Höhendifferenzen von Karböden gegenüber schichtparalleler Abdachung der Gipfelfirste ergeben sich Hinweise über die Dauer der Karbonatlösung. Die auf kaum geneigter Fläche in analoger Höhenlage in den Schwyzer Alpen ermittelten $0,015 \mathrm{~mm} / \mathrm{a}$ sind auf stärker geneigter Unterlage, wo nicht ein Teil des gelösten $\mathrm{CaCO}_{3}$ auf flachen Karsttreppen durch Verdunstung gleich wieder ausfällt, also vor allem an Karwänden, zu gering. Selbst bei Annahme von $0,1 \mathrm{~mm} / \mathrm{a}$ Unterschied zwischen Karbonat-Lösung auf der Gipfel-Abdachung und in der Karwanne ergäben sich für die Bildung der 250-300 m tiefen Wannen 2,5-3 M a. Dabei dürfte die Lösung in den Kaltzeiten, in denen das Taleis bis zum Aufschwung der Firste reichte, infolge geringen Schmelzwasser-Anfalles recht bescheiden gewesen sein. Zugleich war die kolkende, dynamisch wirksame Ausräumung durch die gegenseitig sich stauenden Eismassen reduziert. In den Warmzeiten dagegen waren die Zungenbecken der kaltzeitlichen Churfirsten-Gletscher bewaldet und die Karbonatlösung durch Huminsäuren gesamthaft erhöht. Die Karbonatlösung der Pflanzendecke war eher bescheiden. Dies belegen unter Moosdecken erhaltene Felszeichnungen auf sandig-kalkigen Bündnerschiefer-Rundhöckern auf Carschenna oberhalb von Thusis GR. Für die Ausräumung der Kare verblieben so nur die relativ kurzen Vorstoßund Abschmelzphasen, die eher kühleren Abschnitte der Warmzeiten sowie ihre wärmsten Phasen.

Die Anfänge der Karbildung würden so mindestens tief ins Pliozän zurückreichen; sie setzten wohl schon unmittelbar nach der Platznahme der Decke, in Kühlphasen an der Miozän/Pliozän-Wende, vor rund $5 \mathrm{M}$ a, ein. Dies ergäbe eine von den Kaltzei- ten bis in die wärmsten Abschnitte der nächsten Warmzeit gemittelte Lösungsrate von 0,05-0,06 $\mathrm{mm} / \mathrm{a}$.

Auf Garschella, zwischen Chäserrugg und Gamser Rugg, ist der Seewer Kalk bis auf die Garschella-Formation (= Gault; FÖLLMI \& OUWEHAND 1987) erodiert worden. Eis floß bis ins Spätwürm vom Tristelcholben-Rosenboden-Chäserugg gegen $\mathrm{N}$ über die Gamsalp ins Becken der Schwendiseen und gegen E über Schlawitz zur Voralp ab. Vom Sicherchamm, vom Gamsberg und vom Sichli-Chli Fulfirst stießen Zungen ins Becken der Voralp und formten Talfurchen und Kleinrelief (Karte).

Die isolierte Kuppe des Gamser Rugg mit Garschella-Formation und Seewer Kalk und die mit Amdener Mergeln gefüllte Senke von GamperfinVoralp sind auf Strukturen des Untergrundes zurückzuführen, der Gamser Rugg auf die nordöstliche Fortsetzung der flachen Aufwölbung über dem Jura-Gewölbe der Lüsis-Schuppe, die auf Palis kulminiert.

Wie im Alpstein im Sax-Schwende-Bruch und in der Gamplüt-Störung zeichnen sich auch im Churfirsten-Alvier-Gebiet schon in den letzten Phasen der Platznahme entstandene Störungen ab, besonders in der verscherten Mulde des Sichelchamm. Diese fällt axial gegen ENE ins Rheintal ab und ließ die Voralp-Walchenbach-Talung entstehen. Fast N-S verlaufen markante Störungen $\mathrm{E}$ des Chäserrugg und $E$ von Gamperfin; durch sie wird der Schenkel der gegen E ausflachenden Wildhauser Mulde staffelartig gegen das Rheintal abgesenkt (Karte). Parallel und senkrecht zum Rheintal verlaufende Bruchsysteme stellen sich zwischen Wartau und Buchs ein. Durch sie wird das Achsengefälle der Alvier-Kreide verringert. Die Störung im unteren Seveler Bach hat die verscherte Platte des Gretschinser Holzes emporgestaucht. Sie paust in der Kreide-Abfolge die im Jura-Stockwerk erfolgte Aufschiebung der Tschuggen- auf die StrahleggSchuppe durch. Die Rinnen zwischen Chäserrugg und Gamperfin - so die auf $1465 \mathrm{~m}$ einsetzende Rossboden-Rinne - wurden vorab subglaziär ausgeräumt. Die warmzeitliche Vertiefung dürfte - infolge der höher reichenden Bewaldung - bescheiden gewesen sein.

\section{Das Rhein-Eis in den riß- und würmzeitlichen Maximalständen}

In den größten Vereisungen stand der Rhein-Gletscher zwischen Sargans und Buchs - wie höchste Überprägungen auf Graten erkennen lassen - zwischen 1900 und $1750 \mathrm{~m}$ (HANTKE \& SEITTER 1985). An der Diffluenz der Gauschla SE des Alvier reichte er bis auf über $1850 \mathrm{~m}$ empor (HANTKE in SEITTER 1988). Dabei scheint jedoch der Eisteiler dem anbrandenden Rhein-Gletscher nicht durch alle Kalt- 
zeiten standgehalten zu haben. Er dürfte - teils schon beim Abschmelzen des riß-, vor allem aber des würmzeitlichen Eises - als Bergsturz von Oberschan niedergebrochen sein. Der ESE vorgelagerte Trunachopf ist an vorgezeichneter Bruchstaffel zerbrochen und zum Absturz bereit.

Aus dem Alvier- und Voralp-Gebiet empfing der Rhein-Gletscher Zuschüsse. Über den Sattel von Wildhaus $(1028 \mathrm{~m})$ verlor er Eis ins oberste Toggenburg. Doch schon bei Unterwasser stauten Säntisthur- und Churfirsten-Gletscher das eingedrungene Rhein-Eis zurück, so da $\beta$ es kaum weiter thurabwärts gelangen konnte (FREY 1916, HANTKE 1967). Über dem Sattel von Wildhaus stand es zur Riß-Eiszeit um $1700 \mathrm{~m}$, zur Würm-Eiszeit um $1500 \mathrm{~m}$ (HANTKE 1970, 1980).

Da der rißzeitliche Rhein-Gletscher an der Gauschla nur wenig über $1850 \mathrm{~m}$ Höhe emporreichte, konnte kein Eis aus der Seez-Walensee-Talung durch die 2050-1950 m hohen Churfirsten-Lücken ins Toggenburg überfließen. Dagegen könnte dies in früheren Kaltzeiten, in denen das Eis nur wenig hinter den mindel- und rißzeitlichen Höchstständen lag und der Hochstau der helvetischen Kalkalpen allenfalls noch geringer war, möglich gewesen sein. Dann müßte sich die spätere En-bloc-Hebung - wie am Südalpen-Rand - um mindestens $250-300 \mathrm{~m}$ bewegt haben. Immerhin dürfte das Rhein-Eis in den größten Kaltzeiten noch fast auf den $1836 \mathrm{~m}$ hohen Sattel NW des Sichelchamm gereicht haben.

Im Alvier-Gebiet floß in den Hochglazialen SisitzEis über den Sisitzgrat $(2016 \mathrm{~m})$ und den Sattel des Margelchopf (P. 1994) ins Quellgebiet des Studner Bachs (Karte). In der Würm-Eiszeit dürfte es im Ried und auf Sisitzsäß erneut auf $200 \mathrm{~m}$ angewachsen sein.

Der Margelchopf ist bis zum Felsaufschwung auf $2100 \mathrm{~m}$ und der Chapf (2048 m) gar bis zur Gipfelkuppe eisüberprägt worden.

\section{Das Relief im Sattel von Wildhaus im letzten Interglazial}

Schon im letzten Interglazial zeigte der $\mathrm{Pa}$ von Wildhaus seine heutige Gestalt. Dies belegen frühwürmzeitliche Ablagerungen $\mathrm{SE}$ von Wildhaus (HEIM \& GAMS 1918) und riß/würm-interglaziale Sedimente S vom Schönenboden (746712/229830/ 1080, WELTEN 1987). Darnach erfolgte dort keine Ausräumung, vielmehr wurde aufgeschüttet. Da auch in früheren Kaltzeiten ins oberste Toggenburg übergeflossenes Rhein-Eis durch Säntisthur- und Churfirsten-Eis gestaut wurde, muß sich auch die frühere glazigene Ausräumung in Grenzen gehalten haben. Die Tiefe des von Eis ausgeräumten Beckens von Unterwasser dürfte sich zwischen 50 und $100 \mathrm{~m}$ bewegen. In der Wildhauser Mulde stehen zudem die jüngsten Gesteine an (FORRER 1948, HERB 1962. 1967). Südlich von Alt St. Johann und Unterwasser und im Becken der Schwendiseen haben die wiederholt vorgestoßenen Churfirsten-Gletscher über $50 \mathrm{~m}$ tiefe Zungenbecken ausgekolkt (ANGEHRN, schr. Mitt.). Die glazigene Ausräumung dürfte weit zurückreichen, da die Kolk-Raten in tiefer gelegenen und flacheren Gebieten eher wieder geringer werden.

\section{Die frühen spätwürmzeitlichen Gletscherstände}

Im Stadium von Konstanz (= Hurden-Rapperswil) reichte der Rhein-Gletscher über den Sattel von Wildhaus, vereinigte sich mit Säntisthur- und Churfirsten-Eis und endete als Thur-Gletscher bei Krummenau. Seitenmoränen mehrerer Staffeln sind W und $\mathrm{S}$ von Nesslau angedeutet. Beim weiteren Abschmelzen riß die Verbindung von Rhein- und ThurEis ab.

Im Stadium von Koblach (= Ziegelbrücke) lag der Sattel von Wildhaus noch unterThur-Eis. Dieses endete gegen E wenig unterhalb des Dorfes, gegen W vor Starkenbach. Der Tesel-Gletscher von Altmann undWildhuser Schafberg stirnte im Vorfeld des auch gegen $\mathrm{E}$ abfließenden Toggenburger Eises. Sein bedeutendster Lieferant, der Säntisthur-Gletscher, wurde bis zum Rappen-Gletscher aus dem Kar zwischen Frümsel und Selun von Churfirsten-Eis unterstützt. Dieser letzte Churfirsten-Gletscher spaltete sich - durch SSW-NNE verlaufende Brüche etwas kanalisiert - kurz vor Erreichen derTalsohle in zwei Zungen auf. Von NE vermochte der Leist-Gletscher - dank Zuschüssen zwischen Selun und Glattchamm - ebenfalls bis gegen Starkenbach vorzustoBen. Von der Nord-Abdachung des Häderenberg hingen Firne in mehreren Zungen bis fast ins Thurtal herab (Karte).

Bis ins Stadium von Feldkirch (= Weesen) ist das Toggenburger Eis etwas zurückgeschmolzen, was interner gelegene Wälle bekunden. Deutlicher manifestiert sich das Zurückschmelzen bei den großen Talgletschern, die bei Feldkirch bzw. Weesen endeten. Dies hängt wohl mit den aus dem Walgau und aus dem Linthtal austretenden Schmelzwässern zusammen, deren Gletscher an den Ausgängen endeten.

Der Voralp-Gletscher erreichte noch den RheinGletscher, was eine Mittelmoräne SWvon Grabs belegt. Zugehörige Seitenmoränen lassen sich bei Litten erkennen (HANTKE 1970).

Im Stadium von Sargans sind Säntisthur- und Churfirsten-Gletscher selbständig geworden, was zwei schuttarme, rasch sich folgende Endmoränen bekunden. Entsprechende Stände sind beim VoralpGletscher im vorderen Walchenbachtal durch stirnnahe Moränen angedeutet. 


\section{Der Bergsturz der Voralp}

Die im NW-Abbruch des Chapf zwischen Schlößlichopf und Spitzigi Chöpf fehlende Gesteinsmasse - Druesberg-Schichten, Schrattenkalk und Garschella-Formation - liegen nur zum Teil im Trümmerfeld des prähistorischen Voralp-Bergsturzes vor. Dieses bedeckt im Raum Chalchofen-Unter Stafel-Höhi-Lidmäl-Guferen rund $0,7 \mathrm{~km}^{2}$ und staute Naus- und Langgner Bach zum 25 ha großen Voralpsee. Bei einer mittleren Mächtigkeit von $40 \mathrm{~m}$ ergäbe sich ein Volumen der Trümmermasse von $2500000 \mathrm{~m}^{3}$. Ihr Niederbrechen setzte spätestens im Spätwürm ein, findet sich doch unter den Sturzmassen noch Moräne. Da diese noch nicht dem Fels aufliegt, dürfte der Sturz mehrphasig erfolgt sein: ein älterer mag bis ins Präwürm zurückreichen; jedenfalls scheinen sich die Stürze in verschiedenen Abschnitten der Würm-Eiszeit ereignet zu haben.

Der Blockschuttfächer von Inggarnascht mit Blökken von Kieselkalk, Druesberg-Schichten und Schrattenkalk ist in einer letzten spätwürmzeitlichen Abbruchphase niedergefahren.

\section{Spätwürm-Moränen auf der NE-Seite der Churfirsten-Alvier-Kette}

Auffällige Moränen des Spätwürms liegen S derVoralp, auf Sisitz. In einem älteren Stand nahm der Sisitz-Gletscher noch das Zungenbecken des Ried ein. Ein innerer Stand wird am W-Hang des SisitzGrates durch Seitenmoränen belegt. Weiter N umschließt eine hufeisenförmige Stirnmoräne ein kleines Zungenbecken. Dieses ist bis weit in den Sommer hinein mit Schnee, später mit Schmelzwasser gefüllt. Bei Bisenlagen wird Schnee über den SisitzGrat geweht, was sich schon im Spätwürm ereignet haben dürfte.

Auf Schlawitz liegen Pakete von vermergeltem Schrattenkalk; sie sind im Spätwürm auf Eis niedergebrochen und abgeglitten.

Höchste Moränen liegen in den Churfirsten vor Nbis E-exponierten Karen. Einzugsgebiete und Zungenenden unterscheiden sich etwas in ihrer Höhenlage, sind aber demselben Stadium zuzuordnen. Differenzen bekunden Unterschiede in Schnee-Akkumulation und Firn-Erhaltung.

\section{Spätwürm-Moränen auf der S- und SW-Seite von Churfirsten und Alvier}

Markante Spätwürm-Moränen haben sich auf der Sund SW-Seite der Churfirsten-Alvier-Kette erhal- ten. Sie bekunden, daß der Schnee an Steilflanken jeweils rasch abglitt, frostgesprengtes Gesteinsgut mitriß und in den Alpkesseln - wie heute weit bescheidener der Lawinenschnee - sich sammelte, umkristallisierte und als Eiszungen sich gegen das Seeztal bewegte.

Bis ins Stadium von Weesen lieferten Schneemassen von den Schären, vom Nägeliberg und vom Leistchamm dem Walensee-Arm des Rhein-Gletschers letzte Zuschüsse. Im Stadium von Sargans kalbte die Zunge des Leistchamm-Gletschers bei Quinten in den vor der Front des Rhein-Gletschers sich bildenden Walensee, der vermutlich bis gegen Sargans reichte. Die vom Nägeliberg herabhängende Eiszunge schüttete einen Sanderkegel in den See.

\section{Jüngsttertiäre Florenrelikte}

Wie im Alpstein (hANTKE \& SEITTER 1985, SEITTER 1988f.) hat sich auch in der Churfirsten-AlvierKette über den Rhein-Gletscherarmen und unter feuchtigkeitsspendenden Firnkappen an steilen SLagen eine die Eiszeiten überdauernde jüngsttertiäre Reliktflora erhalten. Dabei dürfte die Artenzahl - trotz der um 150 m höheren Reichweite des Rhein-Eises und der um 160 m niedrigeren Berge kaum bescheidener gewesen sein als im Alpstein. Während sich dort eine Reliktflora über nahezu $1000 \mathrm{~m}$ Höhendifferenz entfalten konnte, war diese in den Churfristen auf $600 \mathrm{~m}$ und am Alvier auf nur $480 \mathrm{~m}$ beschränkt. Doch dürften wegen der hohen sommerlichen Sonnenstände über den Kaltluftströmen des Rhein-Gletschers an den Churfirsten über 200 und im Alvier-Gebiet noch über 150 Arten die Eiszeiten überdauert haben (SEITTER 1988f.).

\section{Zusammenfassung}

Im verkarsteten Churfirsten-Alvier-Gebiet zeigt sich, daß tektonische Vorzeichnungen die Talbildung bis in Einzelheiten bestimmen und glaziäre und subglaziäre Schmelzwässer das weitere erosive Geschehen gefördert haben. Zwischen den Churfirsten-Gipfeln haben sich seit dem Pliozän Kare gebildet; bei vielen ist die Rückwand durchgebrochen. In den größten Kaltzeiten reichte der bei Sargans sich aufspaltende Rhein-Gletscher bis $150 \mathrm{~m}$ unter den niedrigsten Churfirsten-Sattel. Falls je Eis aus dem Walenseetal ins Toggenburg übergeflossen ist, kann dies nur bei hohen Ständen und schon niedergebrochenen Kar-Rückwänden im Jungpliozän bis Altpleistozän geschehen sein. Dies würde - wie am Südalpen-Rand - eine spätere En-bloc-Hebung um $300 \mathrm{~m}$ erfordern. 
Der Abtrag ging differenziert vor sich. Wo Spannungen den Gesteinsverband gelockert haben, ist er, vorab zu Beginn der Kaltzeiten - und solche reichen vor das Pleistozän zurück - bedeutend gewesen. In Warmzeiten mit dichter Bestockung und hoher Waldgrenze dagegen war selbst der Lösungsabtrag bescheiden. Wie im Alpstein haben in der Churfirsten-Alvier-Kette in S-bis W-Lagen tertiäre Florenrelikte die Eiszeiten überdauert.

Dank schulde ich lieben Kollegen, die mich bei der Feldarbeit begleitet und meine Gedankengänge kritisch nachvollzogen haben, vorab den Herren Proff. Dr. W. STUMm und H. HEIERLI. Herrn Dr. P. ANGEHRN, Degersheim, verdanke ich Bohrtiefen aus dem Obertoggenburg. Die Durchsicht des Manuskriptes übernahmen die Herren Drs. K. BÄCHTIGER und A. EGLI, jene des Summary Herr Dr. J.-P. BECKMANN.

\section{Geologische Karten}

BRIEGEL, U. (1972): Geologische Karte der Alvier-Gruppe 1: 10 000, Dep. Geol. Landesaufn., Basel.

ESCHER v. d. Linth, A. (1869): Geognostische Karte der St. Galler und Appenzeller Alpen 1:25 000. Orig. verschollen, Kopie von ERNST MÜNSTER im Auftrag der Regierung des Kts. St. Gallen, Staatsarchiv St. Gallen.

- et al. (1875): Blatt IX Schwyz-Glarus-Appenzell-Sargans, Geol. Karte Schweiz 1:100 000, Schweiz. geol. Komm.

HANTKE, R. (1970, 1980): Das Gebiet zwischen Linth und Rhein zur Würm-Eiszeit 1:100 000. Vjschr. natf. Ges. Zürich 115/1; Eiszeitalter 2, Karte 1.

HEIM. ARN., \& OBERHOLZER, J. (1907): Geologische Karte der Gebirge am Walensee 1:25 000. Geol. Spez.-Karte 44, Schweiz. geol. Komm.

- , \& - (1917): Geologische Karte der Alvier-Gruppe 1:25 000. Geol. Spez.-Karte 80, Schweiz. geol. Komm.

HERB, R. (1962): Geologische Karte der Gulmengruppe und der Amdener Höhen 1:15000. In: Geologie von Amden, Beitr. geol. Karte Schweiz NF 114.

OBERHOLZER, J., et al. (1942, 1984): Geologische Karte des Kantons Glarus 1:50000. Geol. Spez.-Karte 117, Schweiz. geol. Komm. (1980: Neudruck).

THIERSTEIN, H. R. (1969): Geologische Karte der Voralp (Gemeinde Grabs SG) 1:10 000. In: Dipl.-Arb. Phil. Fak. II Univ. Zürich, Dep. Geol. Inst. ETH, Zürich.

\section{Literatur}

BRIEGEL, U. (1972): Geologie der östlichen Alviergruppe (helvetische Decken der Ostschweiz) unter besonderer Berücksichtigung der Drusberg- und Schrattenkalkformation (Unterkreide). Eclogae geol. Helv. 65/2: 425-483.

DIEGEL, F. (1973): Zur Korrelation der helvetischen Zementsteinschichten zwischen Glärnisch und Alvier. Diss. Phil. Fak. II, Univ. Zürich.

FÖLLMI, K. B., \& OUWEHAND, P. J. (1987): Garschella-Formation und Götzis-Schichten (Aptian-Coniacian): Neue stratigraphische Daten aus dem Helvetikum der Ostschweiz und des Vorarlbergs. Eclogae geol. Helv. 80/1.

FORRER, M. (1948): Zur Geologie der östlichen Wildhauser Mulde. Jb. st. gall. natw. Ges. 73: 1-97.

FREY, A. P. (1916): Die Vergletscherung des obern Thurgebietes. Jb. st. gall. natw. Ges. 54 (1914-16): 35-131.

FUNK, H., et al. (1987): Erläuterungen zu Blatt 1115 Säntis Geol. Atlas Schweiz 1:25 000. Geol. Landesaufn.

GREBER, E. (1987): Die Geologie der mittleren Churfirsten unter besonderer Berücksichtigung von "mittel" kretazischen Spaltenfüllungen an der Grenze Schrattenkalk/Garschella-Formation. Dipl.-Arb. Abt. Naturw. ETH Zürich, Dep. Geol. Inst. ETH.

GUTZWILLER, A. (1873): Das Verbreitungsgebiet des Sentisgletschers zur Eiszeit. Ber. Thätigk. natw. Ges. St. Gallen 1871/72: 80-155.

HANTKE, R. (1961): Tektonik der helvetischen Kalkalpen zwischen Obwalden und dem St. Galler Rheintal. Vjschr. natf. Ges. Zürich 106/1: 1-212.

- (1967): Die würmeiszeitliche Vergletscherung im oberen Toggenburg (Kt. St. Gallen). Vjschr. natf. Ges. Zürich 112/4: 223-242.

- (1970): Die Diffluenz des würmeiszeitlichen Rheingletschers bei Sargans und die spätglazialen Gletscherstände in der Walensee-Talung und im Rheintal. Vjschr. natf. Ges. Zürich 115/1: 101-126.

- (1980): Eiszeitalter, Band 2, Thun.

- (1982): Zur Talgeschichte des Gebietes zwischen Pragelund Klausenpaß. Ber. schwyz. natf. Ges. 8: 3-12, Einsiedeln.

- (1987): Zur jungtertiären Geschichte des Alpenrheintales. Mitt. österr. geol. Ges., im Druck.

- (1988): Zur Geologie der Kantone St. Gallen und Appenzell. In: SEITTER, H.: Flora der Kantone St. Gallen und beider Appenzell 1, St. Gallen-Rorschach.

- \& SEITTER, H. (1985): Vermochten an nie vereisten Lagen im Alpstein jüngsttertiäre Florenrelikte die Eiszeiten zu überdauern? Ber. st. gall. natw. Ges. 82: 55-107.

HEIERLI, H. (1984): Ostschweizer Alpen und ihr Vorland Säntismassiv, Churfirsten, Mattstock, Alviergruppe, Appenzeller Molasse. Samml. geol. Führer 75 - Berlin, Stuttgart.

HEIM, ALB. (1891): Geologie der Hochalpen zwischen Reuss und Rhein. Beitr. geol. Karte Schweiz 25.

- (1919, 1921): Geologie der Schweiz 1, 2/1, Leipzig.

- , et al. (1905): Das Säntisgebirge. Beitr. geol. Karte Schweiz NF 16.

HEIM, ARN. (1917): Tektonik und Oberflächengestaltung Monographie der Churfirsten-Mattstock-Gruppe 4. Beitr. geol. Karte Schweiz NF 20/4. 
- \& GAMS, H. (1918): Interglaziale Bildungen bei Wildhaus. Vjschr. natf. Ges. Zürich 63/1-2: 19-33.

HELBLING, R. et al. (1938): Zur Tektonik des St. Galler Oberlandes. Beitr. geol. Karte Schweiz NF 76/2.

HERB, R. (1962): Geologie von Amden unter besonderer Berücksichtigung der Flyschbildungen. Beitr. geol. Karte Schweiz NF 114.

- (1967): Abschnitt II: Stein-Wildhaus-Buchs. Geol. Führer Schweiz 8: 704-712.

KEMPF, T. A. (1966): Geologie des westlichen Säntisgebirges. Beitr. geol. Karte Schweiz NF 128.

LUDWIG, A. (1894): In der Churfirsten-Alvierkette - Thätigk. Ber. st. gall. natw. Ges. (1892/93): 331-371.

- (1897): Die Alviergruppe. Ber. Thätigk. st. gall. natw. Ges. (1895/96): 294-363.

- (1912): Über die Vorgänge bei der Talbildung. Eclogae geol. Helv. 12/2: 245-264.
- (1919): Über Talbildung in den Alpen und im Vorland. Jb. SAC 53 (1918): 225-244.

MAURER, E. (1952): Zum Problem der Talgeschichte des obersten Toggenburgs während der späteren Eiszeit. Dipl.Arb. Phil. Fak. II Univ. Zürich, Dep. Geol. Inst. ETH Zürich.

MOESCH, C. (1881): Geologische Beschreibung der Kalkstein- und Schiefergebirge der Kantone Appenzell, St. GalIen, Glarus und Schwyz (BI. IX). Beitr. geol. Karte Schweiz $14 / 3$.

SEITTER, H. (1987f.): Flora der Kantone St. Gallen und beider Appenzell. St. Gallen-Rorschach.

THIERSTEIN, H. R. (1969): Geologisch-mikropaläontologische Untersuchungen in der Grabser Voralp. Dipl.-Arb. Phil. Fak. II Univ. Zürich, Dep. Geol. Inst. ETH, Zürich.

WELTEN, M. (1987): Neue pollenanalytische Ergebnisse über das jüngere Quartär der nordalpinen Schweiz. Beitr. geol. Karte Schweiz im Druck. 